\title{
Uncommon gastrointestinal bleeding during targeted therapy for advanced renal cell carcinoma: A report of four cases
}

\author{
SHINTARO FUJIHARA ${ }^{1}$, HIROHITO MORI ${ }^{1}$, HIDEKI KOBARA ${ }^{1}$, NORIKO NISHIYAMA ${ }^{1}$, MAKI AYAKI ${ }^{1}$, \\ RYO OHATA $^{2}$, NOBUFUMI UEDA ${ }^{3}$, MIKIO SUGIMOTO ${ }^{3}$, YOSHIYUKI KAKEHI ${ }^{3}$ and TSUTOMU MASAKI $^{1}$ \\ ${ }^{1}$ Department of Gastroenterology and Neurology, Kagawa University Faculty of Medicine, Kagawa 761-0793; \\ ${ }^{2}$ Department of Urology, Tottori Red Cross Hospital, Tottori 680-8517; ${ }^{3}$ Department of Urology, \\ Faculty of Medicine, Kagawa University, Kagawa 761-0793, Japan
}

Received September 26, 2014; Accepted June 16, 2015

DOI: $10.3892 / \mathrm{ol} .2015 .3671$

\begin{abstract}
Clinically available targeted agents to treat advanced renal cell carcinoma (RCC) include sunitinib, sorafenib and temsirolimus. Sorafenib and sunitinib have been associated with bleeding in selected trials, but clinical and endoscopic characteristics of gastrointestinal bleeding are not well described. Herein, we report four cases of advanced RCC in which endoscopic hemostasis effectively resolved high-grade, life-threatening gastrointestinal bleeding that occurred during targeted therapy. Although stomatitis and mucositis have occurred during targeted therapies, life-threatening gastrointestinal bleeding is less common. In these four patients, the origins of gastrointestinal bleeding were identified, and complete endoscopic hemostasis was achieved. Endoscopies revealed variable characteristics including angiodysplasia, multiple gastric ulcers and oozing bleeding of the normal mucosa. Although the most effective diagnostic and treatment strategies are disputed, endoscopic examinations are best performed before starting targeted therapies. Additionally, these patients should be monitored even for rare life-threatening events.
\end{abstract}

\section{Introduction}

Renal cell carcinoma ( $R C C$ ) is diagnosed in $\sim 340,000$ individuals worldwide annually, resulting in 143,000 mortalities (1). Numerous patients present with advanced or unresectable disease (2). RCC is often resistant to chemotherapy and cytokines including interleukin 2 and interferon- $\alpha$ (3). The recent advent of targeted agents for RCC has led to continuous

Correspondence to: Professor Tsutomu Masaki, Department of Gastroenterology and Neurology, Kagawa University Faculty of Medicine, 1750-1 Ikenobe, Miki-cho, Kita-gun, Kagawa 761-0793, Japan

E-mail: tmasaki@med.kagawa-u.ac.jp

Key words: gastrointestinal bleeding, temsirolimus, sunitinib, sorafenib, targeted therapy, argon plasma coagulation treatment for the majority of patients over increasingly long periods of time.

Approved, clinically available targeted agents to treat advanced RCC include the vascular endothelial growth factor receptor (VEGFR) tyrosine kinase inhibitors (TKIs) sunitinib, pazopanib, sorafenib and axitinib, and the mammalian target of rapamycin (mTOR) inhibitor temsirolimus and everolimus (4). These drugs have a great effect in untreated and previously treated patients with advanced RCC (5-7).

However, a new pattern of adverse events has emerged, including bleeding, thrombotic events, hypertension and gastrointestinal tract perforation. Sorafenib and sunitinib in particular have been associated with bleeding in selected trials $(5,6)$. Severe adverse events associated with temsirolimus are uncommon; however, stomatitis and mucositis have occurred during targeted therapy (8), and we previously reported a case of life-threatening gastrointestinal bleeding associated with temsirolimus (9). Major and minor bleeding complications have been reported in targeted therapy for advanced RCC; however, the clinical and endoscopic characteristics of gastrointestinal bleeding are not well described.

Herein, we report a case series with advanced RCC in which endoscopic hemostasis effectively resolved high-grade, life-threatening gastrointestinal bleeding that occurred during targeted therapy. Written informed consent was obtained from the patient and the study was approved by the ethics committee of Kagawa University (Takamatsu, Japan).

\section{Case reports}

Case 1. The patient was a 79-year-old male diagnosed with right RCC (cT3aN2M1) and multiple lung metastases. He received radical right nephrectomy in October 2007. The resected tumor was pathologically diagnosed as clear cell carcinoma, G2, $45 \mathrm{~mm}$. In January 2012, the patient received sunitinib (37.5 mg/day) for metastasis, after which he complained of proteinuria and severe lower back pain. He started axitinib treatment (5 mg/day) in June 2013, during which he complained of severe diarrhea. The sunitinib dose was changed to $50 \mathrm{mg} /$ day in July 2013; 179 days after starting sunitinib therapy, he was admitted to our hospital at Kagawa University Faculty of Medicine for melena. A complete blood count revealed a 


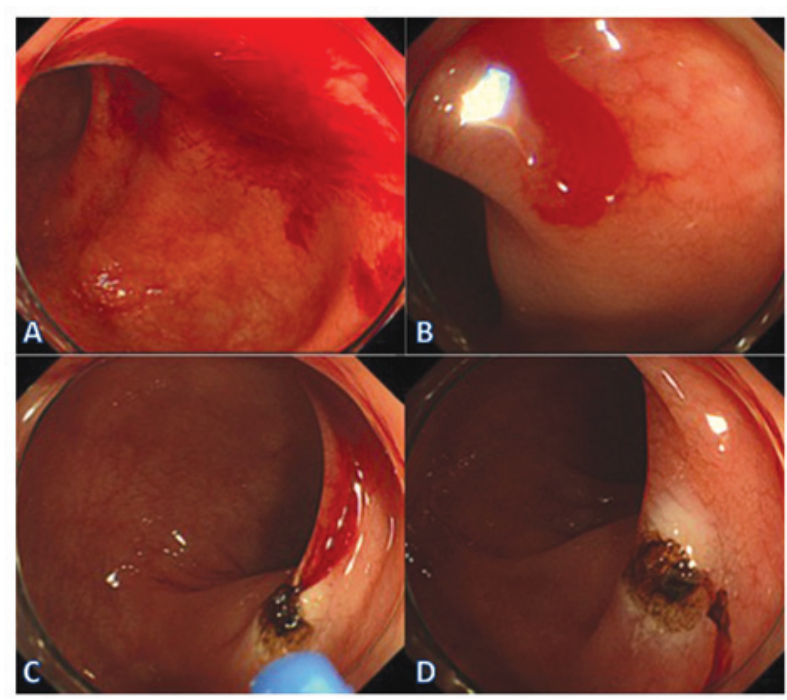

Figure 1. (A) Colonoscopy revealed oozing bleeding of the normal mucosa in the sigmoid colon. (B) After cleaning the colonic mucosa with a water jet, $(\mathrm{C}$ and $\mathrm{D})$ argon plasma coagulation was performed.

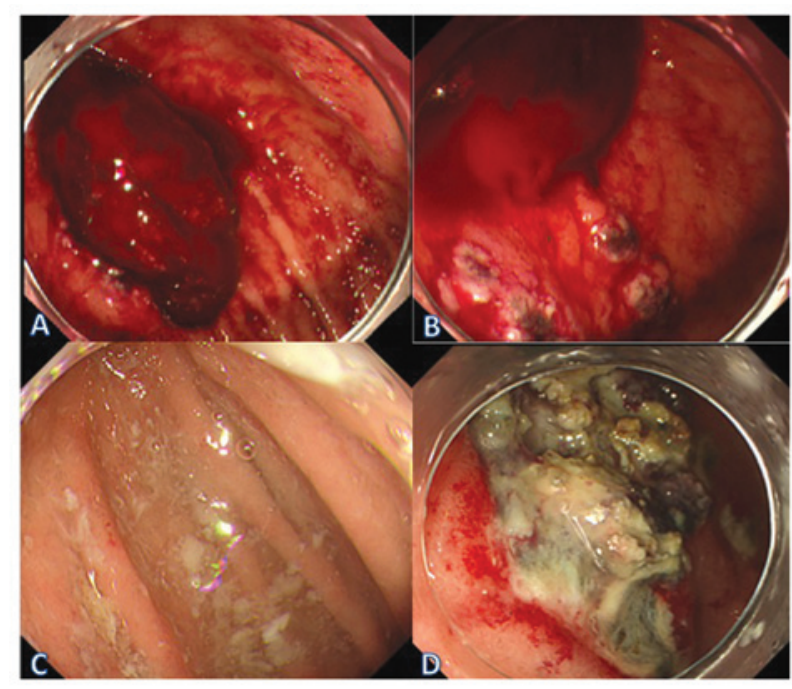

Figure 2. (A) Esophagogastroduodenoscopy revealed fresh blood and a number of clots. (B) After cleaning the gastric mucosa with a water jet, numerous irregular-shaped, non-bleeding gastric ulcers was observed in the fornix. (C) Earlier esophagogastroduodenoscopy before starting targeted therapy. (D) Endoscopic hemostasis with was performed with hemostastic forceps.

marked drop in hemoglobin from $8.9 \mathrm{~g} / \mathrm{dl} 11$ days earlier to $6.1 \mathrm{~g} / \mathrm{dl}$. Colonoscopy revealed oozing bleeding of the normal mucosa (Fig. 1A and B). Endoscopic hemostasis with argon plasma coagulation (APC) was performed (Fig. 1C and D). The patient did not require a blood transfusion following the hemostasis treatment.

Case 2. The patient was a 79-year-old male diagnosed with right RCC (cT3aN2M1) and adrenal gland and lung metastasis. He had a history of gastric cancer. He received a radical right nephrectomy in December 2008. The resected tumor was pathologically diagnosed as clear cell carcinoma, G2>G3 INF $\beta$, $\mathrm{v}(+)$, ly(+) $7 \mathrm{~cm}$ pT3a. In August 2012, a follow-up computed

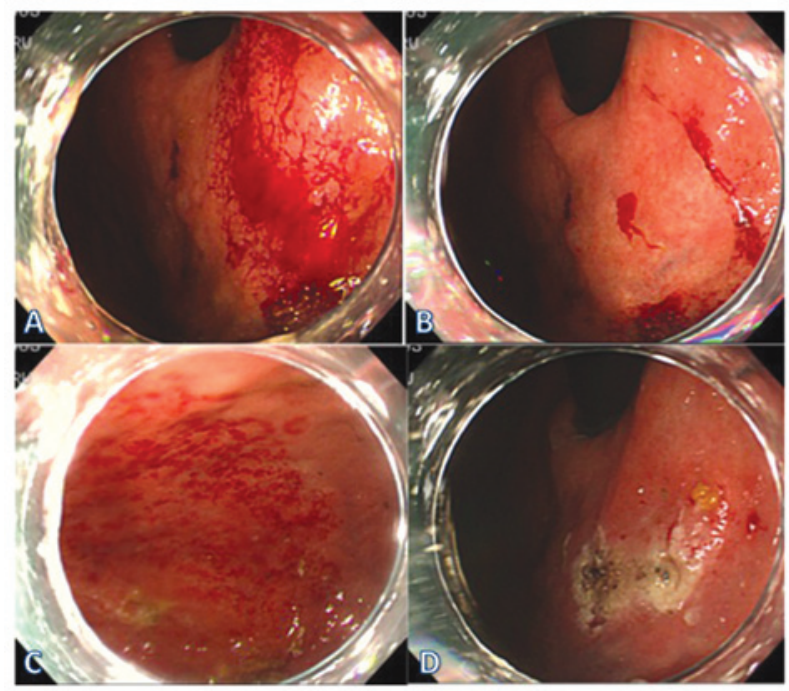

Figure 3. (A) Esophagogastroduodenoscopy revealed oozing bleeding of the upper gastric body that resembled vascular ectasia. (B) After cleaning the gastric mucosa with a water jet, $(C)$ a number of non-bleeding vascular ectasia were observed in the fornix. (D) Argon plasma coagulation was performed.

tomography scan revealed new hepatic metastasis, and the patient received sunitinib treatment (37.5 mg/day). Following the sunitinib therapy, the patient complained of severe fatigue, so his treatment was changed to axitinib (10 mg/day). He contracted drug-induced interstitial pneumonia 20 days after starting axitinib therapy. He was switched to sorafenib (800 mg/day) 25 days after starting sorafenib therapy. As he complained of general fatigue again, the sorafenib dose was decreased to $200 \mathrm{mg} /$ day. He was admitted to our hospital for hematemesis and unconsciousness 73 days after changing the sorafenib dose. A complete blood count revealed that his hemoglobin level had dropped from $12.2 \mathrm{~g} / \mathrm{dl} 3$ days earlier to $8.4 \mathrm{~g} / \mathrm{dl}$. Esophagogastroduodenoscopy (EGD) revealed arterial bleeding with clots from multiple gastric ulcers (Fig. 2A and B). An earlier EGD had revealed no gastric ulcers prior to starting sorafenib (Fig. 2C). Endoscopic hemostasis was performed with hemostatic forceps (Fig. 2D); blood transfusion was not required following the treatment.

Pathological examination revealed atrophic gastritis and intestinal metaplasia with inflammatory cells. Overall, the patient required a one session of endoscopic hemostasis and four units of transfused blood for severe anemia during these 2 weeks. Four weeks after discontinuing sorafenib, the patient's symptoms resolved and he was discharged in good clinical condition 60 days after hospital admission.

Case 3. The patient was a 81-year-old male diagnosed with left RCC (cT3aNOM0). Temsirolimus ( $25 \mathrm{mg} /$ day) was started in January 2011. Approximately 2 months later, the patient contracted drug-induced interstitial pneumonia, and so the drug was stopped.

The patient's blood test results at various follow-up points revealed mild stable anemia (hemoglobin, 10.1-10.7 g/dl), but other laboratory parameters were normal on all occasions. Interstitial pneumonia was improved by prescribing a steroid therapy and stopping temsirolimus. Temsirolimus (15 mg/day) was resumed in April 2012. A complete blood count 54 days 
after starting targeted therapy revealed a notable drop in his hemoglobin levels from $7.0 \mathrm{~g} / \mathrm{dl} 10$ days earlier to $5.5 \mathrm{~g} / \mathrm{dl}$. An EGD was therefore performed, which revealed oozing bleeding in the upper gastric body (Fig. 3A and B), and diffuse reddish spots which resembled vascular ectasia extending from the upper gastric body and fornix (Fig. 3C). Endoscopic hemostasis with hemostatic forceps was performed (Fig. 3D). After one session of endoscopic treatment, the patient did not require a blood transfusion, and his anemia improved. In total, he required one session of endoscopic hemostasis and two units of transfused blood for severe anemia during these 2 weeks. Two weeks after discontinuing temsirolimus, the patient's respiratory symptom worsened; he succumbed 14 days after endoscopic treatment.

Case 4. The patient was a 68-year-old female with left RCC and multiple lung metastases, who received a left nephrectomy in January 2011. The resected tumor was pathologically diagnosed as clear cell carcinoma, G2>G3 pT3a. In November 2011, the patient received sorafenib (400 mg/day) for metastasis. Following the sorafenib therapy, the patient complained of hand-foot syndrome, so sunitinib (50 mg/day) was started in December 2011. During sunitinib therapy, she complained of thrombocytopenia, so the sunitinib dose was decreased to $37.5 \mathrm{mg} /$ day in January 2012. The patient was admitted to our hospital due to melena 67 days after starting sunitinib therapy. A complete blood count revealed a drop in her hemoglobin level from $13.4 \mathrm{~g} / \mathrm{dl} 3$ days earlier to $9.8 \mathrm{~g} / \mathrm{dl}$. Colonoscopy revealed diffuse oozing bleeding of the rectosigmoidal colon wall and stool with fresh blood, but endoscopic hemostasis could not be performed. Although the patient was prescribed intravenous carbazochrome sodium sulfonate hydrate with fasting status, her anemia and melena improved and she was discharged 37 days after discontinuing sunitinib.

\section{Discussion}

Various targeted agents to treat advanced RCC are now approved and in clinical use: the TKIs sunitinib and pazopanib, the multikinase inhibitor sorafenib, the anti-VEGF monoclonal antibody bevacizumab, and the mTOR inhibitors temsirolimus and everolimus (4).

Minor hemorrhagic events are relatively common in patients treated with targeted agents; the most common event reported in patients treated with bevacizumab, sunitinib, temsirolimus and everolimus is epistaxis, which is usually resolved without medical attention. Life-threatening hemorrhagic events are relatively rare. Sorafenib and sunitinib are VEGFR TKIs that mainly target the VEGF pathway by inhibiting VEGF or VEGFR. In clinical trials, a small number of cases of high-grade bleeding in sorafenib- and sunitinib-treated patients was recorded. This event may occur at any time during targeted therapy (5-7). Meta-analysis of bleeding risk with VEGFR TKIs revealed that the incidence of bleeding events (all grades) was 16.7\% (95\% CI, 12.7-21.5), and that of high-grade events was $2.4 \%$ (1.6-3.9). The relative risk of all-grade bleeding events associated with sunitinib and sorafenib was $2.0 \%(1.14-3.19 ; \mathrm{P}=0.015)$. These agents are associated with significantly increased bleeding risk (10).
VEGF is essential in maintaining the architecture and integrity of the microvasculature of endothelial cells. When VEGF signaling is blocked by these agents, the repair and renewal capacity of endothelial cells in response to trauma may be impaired, which increases the risk of bleeding (11).

Temsirolimus is an inhibitor of mTOR kinase, a component of intracellular signaling pathways involved in cell growth and proliferation $(12,13)$. The overall incidences of all-grade and high-grade stomatitis were $44.3 \%$ (CI, 32.1-57.1\%) and $3.2 \%$ (95\% CI, 1.9-5.4\%), respectively. Temsirolimus was associated with significantly increased risk of all-grade stomatitis (relative risk, 11.1; 95\% CI, 5.6-22.0; $\mathrm{P}<0.001)(8)$. The mechanism of stomatitis associated with temsirolimus remains unknown. Inhibitors of mTOR kinase may affect mucosal healing in the stomach and trigger gastritis and gastrointestinal bleeding.

Although stomatitis and mucositis have occurred in association with targeted therapies, life-threatening gastrointestinal bleeding is not uncommon. However, the likelihood of encountering rarer adverse events may increase with longer duration of therapy (14). In our five-case series [including our earlier case report (9)], we were able to find the origin of gastrointestinal bleeding in four patients, in whom complete endoscopic hemostasis was achieved. Two patients (Case 3 and our previous case) suffered shock with severe anemia. The endoscopy revealed variable characteristics including angiodysplasia, multiple gastric ulcers and oozing bleeding of the normal mucosa. APC and hemostatic forceps were effective in the four patients who had oozing bleeding due to angiodysplasia, dilated microvessels and/or gastric ulcers. Of the two patients with oozing bleeding during sunitinib and temsirolimus treatment, the bleeding point in one patient was observed to be in the normal mucosal of the sigmoid colon, and in this patient complete endoscopic hemostasis was achieved using APC alone. Only two patients had undergone gastrointestinal endoscopy prior to starting targeted therapy; the other patients did not receive endoscopic examination. Endoscopic examination might be best performed before starting targeted therapy.

As life-threatening hemorrhagic events are uncommon during targeted therapy for advanced RCC, no consensus exists as to the optimal strategy for their diagnosis and treatment. However, endoscopic examinations appear to be more effective when performed prior to starting targeted therapies. Doctors should be aware of even rare life-threatening events, so that their patients may be monitored to identify any serious conditions.

\section{Acknowledgements}

The authors thank Dr Makoto Oryu for providing technical and editorial assistance.

\section{References}

1. International Agency for Research on Cancer: The GLOBOCAN project: cancer incidence and mortality worldwide in 2013. http://globocan.iarc.fr. Accessed February 21, 2014.

2. Motzer RJ, Bander NH and Nanus DM: Renal-cell carcinoma. N Engl J Med 335: 865-875, 1996.

3. Rini BI, Escudier B, Tomczak P, Kaprin A, Szczylik C, Hutson TE, Michaelson MD, Gorbunova VA, Gore ME, Rusakov IG, et al: Comparative effectiveness of axitinib versus sorafenib in advanced renal cell carcinoma (AXIS): A randomised phase 3 trial. Lancet 378: 1931-1939, 2011. 
4. Eisen T, Sternberg CN, Robert C, Mulders P, Pyle L, Zbinden S, Izzedine $\mathrm{H}$ and Escudier B: Targeted therapies for renal cell carcinoma: review of adverse event management strategies. J Natl Cancer Inst 104: 93-113, 2012.

5. Rini BI: Metastatic renal cell carcinoma: Many treatment options, one patient. J Clin Oncol 27: 3225-3234, 2009.

6. Gore ME, Szczylik C, Porta C, Bracarda S, Bjarnason GA Oudard S, Hariharan S, Lee SH, Haanen J, Castellano D, et al: Safety and efficacy of sunitinib for metastatic renal-cell carcinoma: An expanded-access trial. Lancet Oncol 10: 757-763, 2009.

7. Hudes G, Carducci M, Tomczak P, Dutcher J, Figlin R, Kapoor A Staroslawska E, Sosman J, McDermott D, Bodrogi I, et al; Global ARCC Trial: Temsirolimus, interferon alfa, or both for advanced renal-cell carcinoma. N Engl J Med 356: 2271-2281, 2007.

8. Gomez-Fernandez C, Garden BC, Wu S, Feldman DR and Lacouture ME: The risk of skin rash and stomatitis with the mammalian target of rapamycin inhibitor temsirolimus: A systematic review of the literature and meta-analysis. Eur J Cancer 48: 340-346, 2012.
9. Fujihara S, Mori H, Kobara H, Suenaga T, Hayashida Y, Sugimoto M, Kakehi Y and Masaki T: Life-threatening gastrointestinal bleeding during targeted therapy for advanced renal cell carcinoma: A case report. BMC Nephrol 14: 141, 2013.

10. Je Y, Schutz FA and Choueiri TK: Risk of bleeding with vascular endothelial growth factor receptor tyrosine-kinase inhibitors sunitinib and sorafenib: A systematic review and meta-analysis of clinical trials. Lancet Oncol 10: 967-974, 2009.

11. Kamba T and McDonald DM: Mechanisms of adverse effects of anti-VEGF therapy for cancer. Br J Cancer 96: 1788-1795, 2007.

12. Schmelzle T and Hall MN: TOR, a central controller of cell growth. Cell 103: 253-262, 2000.

13. Fingar DC, Richardson CJ, Tee AR, Cheatham L, Tsou C and Blenis J: mTOR controls cell cycle progression through its cell growth effectors S6K1 and 4E-BP1/eukaryotic translation initiation factor 4E. Mol Cell Biol 24: 200-216, 2004.

14. Kirkali Z: Adverse events from targeted therapies in advanced renal cell carcinoma: The impact on long-term use. BJU Int 107: $1722-1732,2011$. 